\title{
Die Wirbeltierfauna aus dem letzten Interglazial von Lehringen (Niedersachsen, Deutschland)
}

\author{
Carmen Houben*)
}

Houben, C. (2003): Die Wirbeltierfauna aus dem letzten Interglazial von Lehringen (Niedersachsen, Deutschland). - Eiszeitalter und Gegenwart, 52: 25-39; Hannover.

Keywords: Eemian, interglacial, Lehringen, vertebrates, fishes, reptiles, aves, mammals, ecology, climate, European, comparison.

Kurzfassung: Das Fossilmaterial der Fundstelle Lehringen wurde revidiert. Die Fische, Reptilien und Vögel wurden erstmals bearbeitet. Auf Grund der Palynologie kann diese Lokalität stratigraphisch eindeutig dem EemInterglazial zugeordnet werden. Obwohl es sich um eine rein warmzeitliche Fauna handelt, treten auch Steppennashorn und Riesenhirsch auf. Weiterhin wird die Fundstelle im Rahmen der mitteleuropäischen Fauna des letzten Interglazials betrachtet.

[The vertebrate fauna of the last interglacial of Lehringen (Lower Saxony, Germany)]

Abstract: Subject of the work is a revision of the fossil assemblage from the Lehringen location, including a first examination of the contained fish-, reptile-, and avian fauna. Based on palynological investigations the location can definitely be attributed to the Eem-Interglacial. Although the fauna represents a fully interglacial assemblage, narrow-nosed rhinoceros and giant deer also occur. Furthermore the location is investigated within the scope of the central European fauna of the last interglacial.

\section{Einführung}

Lehringen ist eine der klassischen Faunenfundstellen aus dem letzten Interglazial Norddeutschlands, da sowohl Flora wie Fauna dokumentiert sind. Die Fundstelle liegt etwa $12 \mathrm{~km}$ ESE von Verden an der Aller (Niedersachsen) [09 $23^{\prime} \mathrm{E}$, $\left.52^{\circ} 52^{\prime} \mathrm{N}\right]$. Es handelt sich um ein Seekreidevorkommen, einen Süßwassermergel, der zu einer

*) Anschrift der Verfasserin: Dipl.-Geol. C. Houben, Institut für Paläontologie, Nussallee 8, D-53129 Bonn
Reihe ähnlicher Fundstellen im Raum zwischen Weser und Elbe gehört.

Die Hohlform des ehemaligen Lehringer Sees ist wahrscheinlich ein Toteisloch, welches sich über eine Länge von $250 \mathrm{~m}$ und eine Breite von $80 \mathrm{~m}$ erstreckte (Deibel-Rosenbrock 1960). In der Sedimentabfolge ist das Eem-Interglazial überliefert, wie Pollenanalysen bestätigen (JESSEN \& MiLTHERs 1928). Die stratigraphische Abfolge der Schichten beginnt mit einer geringmächtigen Torfschicht, die von bis zu $15 \mathrm{~m}$ mächtigen Mergelschichten überlagert wird und wiederum durch Torf abgeschlossen wird. Damit sind wesentliche Teile des letzten Interglazials belegt. Während des Mergelabbaues wurde 1948 das Skelett eines Waldelefanten (Elephas antiquus) im Verband mit einer Eibenholzlanze und Steinartefakten gefunden. Weitere Aufsammlungen durch AlexAnder Rosenbrock erbrachten eine reichhaltige Fauna und Flora.

Die Mammaliafauna wurde erstmals von SICKENBERG (1969) aufgelistet. Eine Revision konnte diese Faunenliste weitgehend bestätigen und erweitern (Houben 2001). Durch eine Materialdurchsicht konnten die Funde erfasst und in den Rahmen der mitteleuropäischen, eemzeitlichen Faunen gestellt werden. Weiterhin wurden die Reptilien, Vögel und Fische aus Lehringen bearbeitet und in der Faunenliste ergänzt.

Adam (1951), Jacob-Friesen (1956) und Thieme \& VEIL (1985) befassten sich eingehend mit den archäologischen Aspekten des Waldelefanten und den archäologischen Artefaktfunden. Die makroskopische Flora wurde von KRÄUSEL (1955) bearbeitet, der insgesamt 130 Pflanzenarten nachweisen konnte. Steusloff (1954) und Boettger (1954) konnten in der Invertebratenfauna 28 Molluskenarten nachweisen. Die Funde werden derzeit im Historischen Museum Verden aufbewahrt. Die Wirbeltierreste (Abb. 1, Tab. 1) wurden weitgehend nicht stratigraphisch geborgen, weswegen hier keine weitere stratigraphische Untergliederung gegeben werden kann. 


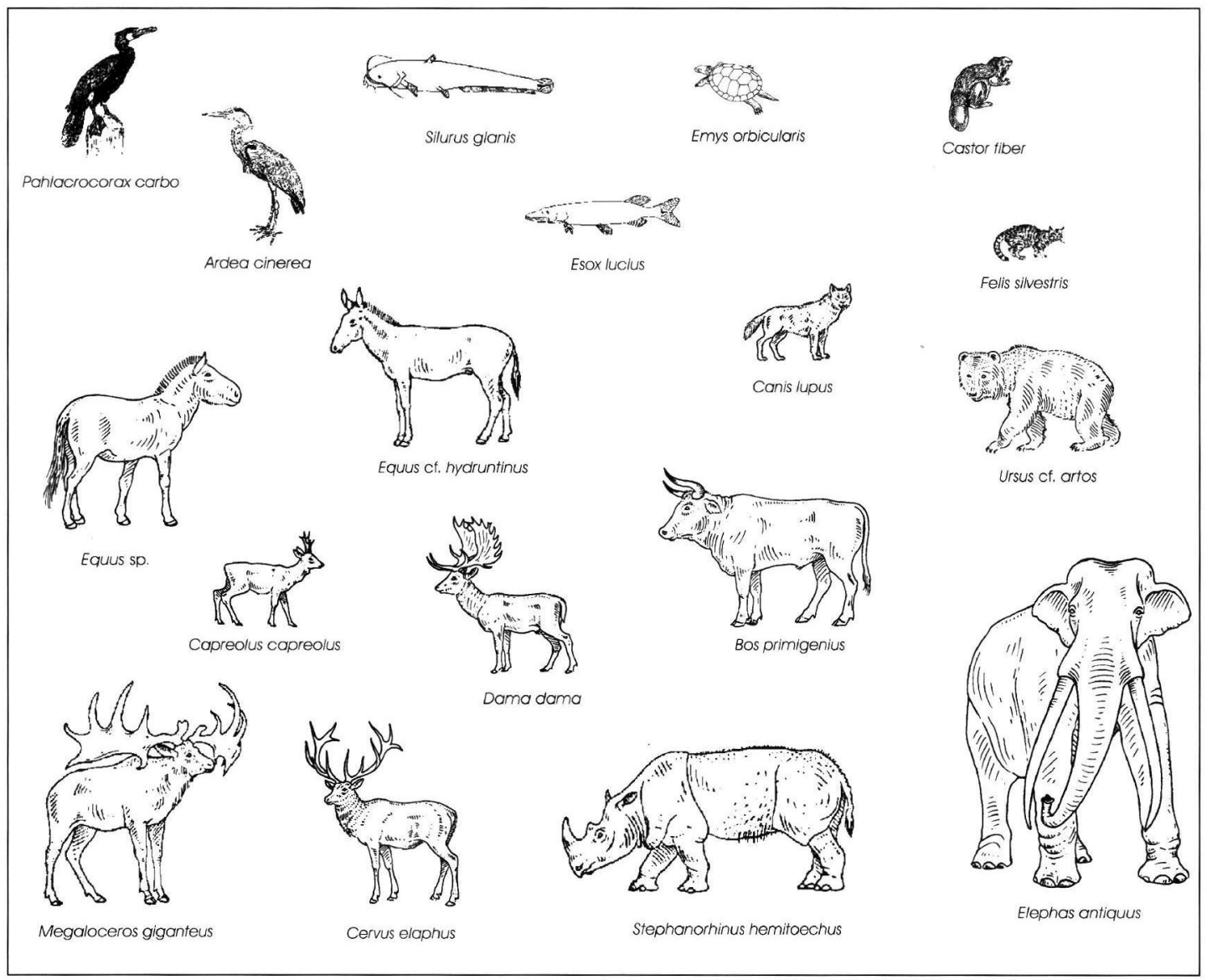

Abb. 1: Graphische Darstellung des Artbestandes aus dem Interglazial von Lehringen (Zeichnungen nach THENIUs).

Fig. 1: Live reconstructions of the different species from the interglacial of the Lehringen location (drawings after THENIUS).

Tab. 1: Systematische Gliederung der Vertebrata.

Tab. 1: Systematic classification of the vertebrates.

\begin{tabular}{|cc|}
\hline Osteichthyes & Proboscidea \\
Esox lucius & Elephas antiquus \\
Silurus glanis & Perissodactyla \\
Reptilia & Equus sp. \\
Emys orbicularis & Equus cf. hydruntinus \\
Aves & Stephanorhinus hemitoechus \\
Phalacrocorax carbo & Artiodactyla \\
Ardea cinerea & Megaloceros giganteus \\
Mammalia & Cervus elaphus \\
Rodentia & Dama dama \\
Castor fiber & Capreolus capreolus \\
Carnivora & Bos primigenius \\
Ursus cf. arctos & \\
Canis lupus & \\
Felis silvestris & \\
\hline
\end{tabular}




\section{Beschreibung der einzelnen Taxa}

\section{Esox lucius Linnaeus, 1758 - Hecht}

Material: 20 Dentalia, 6 Vomeri, 5 Palatina, 10 Ceratohygalia (V 5015.d, V 5040.23-29, V 5040.43-44), 4 Epiphygalia (V 5040.30-33), 6 Opercula (V 5040.34-39), 6 Articularia (V 5040.45-49), 3 Quadrata (V 5040.50-51), 5 Neurocrania, diverse weitere craniale Fragmente und Vertebrae

Vor allem craniales Material von mindestes zehn Individuen von Esox lucius liegt vor. Beeindruckend ist die enorme Größe der Funde. Anhand von rezentem Vergleichsmaterial kann die Größe der Lehringer Individuen auf mindestens 1,00-1,10 m Länge geschätzt werden. Nur zwei Individuen sind deutlich kleiner. Rezent können weibliche Tiere bis zu 1,50 m erreichen, männliche hingegen nicht mehr als $1 \mathrm{~m}$ (Terofal 1984).

E. lucius ist aus mehreren eemzeitlichen Lokalitäten bekannt. In der benachbarten Fundstelle Neddenaverbergen wurde er durch STRUCKMANN (1884) nachgewiesen. Auch in Billstedt-Öjendorf (Hamburg), Phöben, Schönfeld, Klinge, Klinge-Nord, Klein-Lieskow, Tranitz-Süd (alle Brandenburg) und Gröbern (Sachsen-Anhalt) ist der Hecht belegt (BöHME 1997).

Rezente Hechte bevorzugen nährstoffreiche Gewässer von nur wenigen Metern Tiefe mit üppigem, submersem Pflanzenbewuchs sowie reicher Ufervegetation (Müller 1987). Diese Bedingungen müssen auch für den Lehringer See angenommen werden.

\section{Silurus glanis LinNAEUs, 1758 - Wels}

Material: 7 Dentalia-Fragmente, 2 Zahnplatten, 3 Spinae pinnarum pectoralium, 5 Cleithra, diverse weitere Fragmente

Die Mindestindividuenzahl beträgt vier. Zahntragende Elemente sowie Cleithrum und Spinae pinnarum pectoralium erlauben eine genaue $\mathrm{Be}$ stimmung. Die Größe der Lehringer Welse von bis zu $2 \mathrm{~m}$ ist sowohl für das Eem als auch für den rezenten Silurus glanis ungewöhnlich.

MiHÁLIK (1995) beschreibt einen $62 \mathrm{~kg}$ schweren und 2,17 m langen Wels und verweist auf ähnlich große Individuen aus der Elbe, der Moldau und der Luznice. In Deutschland sind Welse über einen Meter jedoch sehr selten. $S$. glanis besitzt ein kontinuierliches Wachstum und kann ein hohes Alter erreichen.
Die enormen Körpermaße weisen auf optimale ökologische Bedingungen im Lehringer See hin.

In eemzeitlichen Fundstellen wurde der Wels in Phöben und Schönfeld (Brandenburg) sowie dem Kleinen Teufelsloch bei Pottstein und dem Fuchsloch bei Siegmannsbrunn (Franken) belegt (BÖHME 1997).

Emys orbicularis (LinNAEUs, 1758) - Sumpfschildkröte (Abb. 2)

Material: 4 Carapax-Fragmente, 22 isolierte Carapax-Platten, 8 Plastron-Fragmente, 8 isolierte Plaston-Platten

Emys orbicularis kann mit mindestens zwölf Tieren in Lehringen nachgewiesen werden. Dabei liegt ein vollständig erhaltener Knochenpanzer (V 5015-16) eines Individuums vor. Die charakteristischen Schildfurchen des Hornpanzers gewähren eine sichere Identifizierung auf Artniveau.

Die Größe der vorhandenen Rückenpanzer liegt zwischen 117 und $140 \mathrm{~mm}$. Rezent erreicht E. orbicularis eine durchschnittliche Länge von $180 \mathrm{~mm}$ bis 200 mm (MLYNARSKI \& WERMUTH 1971). Nach UlLRICH (1984) weisen adulte, jungpleistozäne Sumpfschildkröten eine Carapaxlänge zwischen 150 und $170 \mathrm{~mm}$ auf, wobei die maximale Länge bis $\mathrm{zu} 215 \mathrm{~mm}$ betragen kann. Die Lehringer Stücke liegen damit in ihrer Größe im unteren bis mittleren Streubereich anderer jungpleistozäner Fundstellen wie Burgtonna und Weimar-Stadtgebiet (Thüringen) sowie Cannstatt (Baden-Württemberg) und Schönfeld (UllRich 1984, STRIEGLER 1991). Die geringe Größe der Lehringer Schildkröten beruht eventuell auf ihrem Individualalter. Andererseits ist es durch die unklare Fundlage möglich, dass sie nicht aus den Schichten des Wärmeoptimums stammen, sondern aus Schichten des beginnenden bzw. endenden Eems, und somit kühlere Bedingungen das Wachstum einschränkten.

E. orbicularis ist im Vergleich mit anderen rezenten Schildkröten gegenüber Temperaturschwankungen und größerer Kälte am widerstandsfähigsten und deshalb vermutlich in Europa am weitesten nach Norden vorgedrungen (MLYNARSKI 1969). Die Fortpflanzung und Entwicklung hängt entscheidend von ökologischen Faktoren ab und setzt ein gemäßigtes, relativ kontinentales, mediterran beeinflusstes Klima mit warmen und sonnigen Sommern voraus (STRIEGLER 1991). 


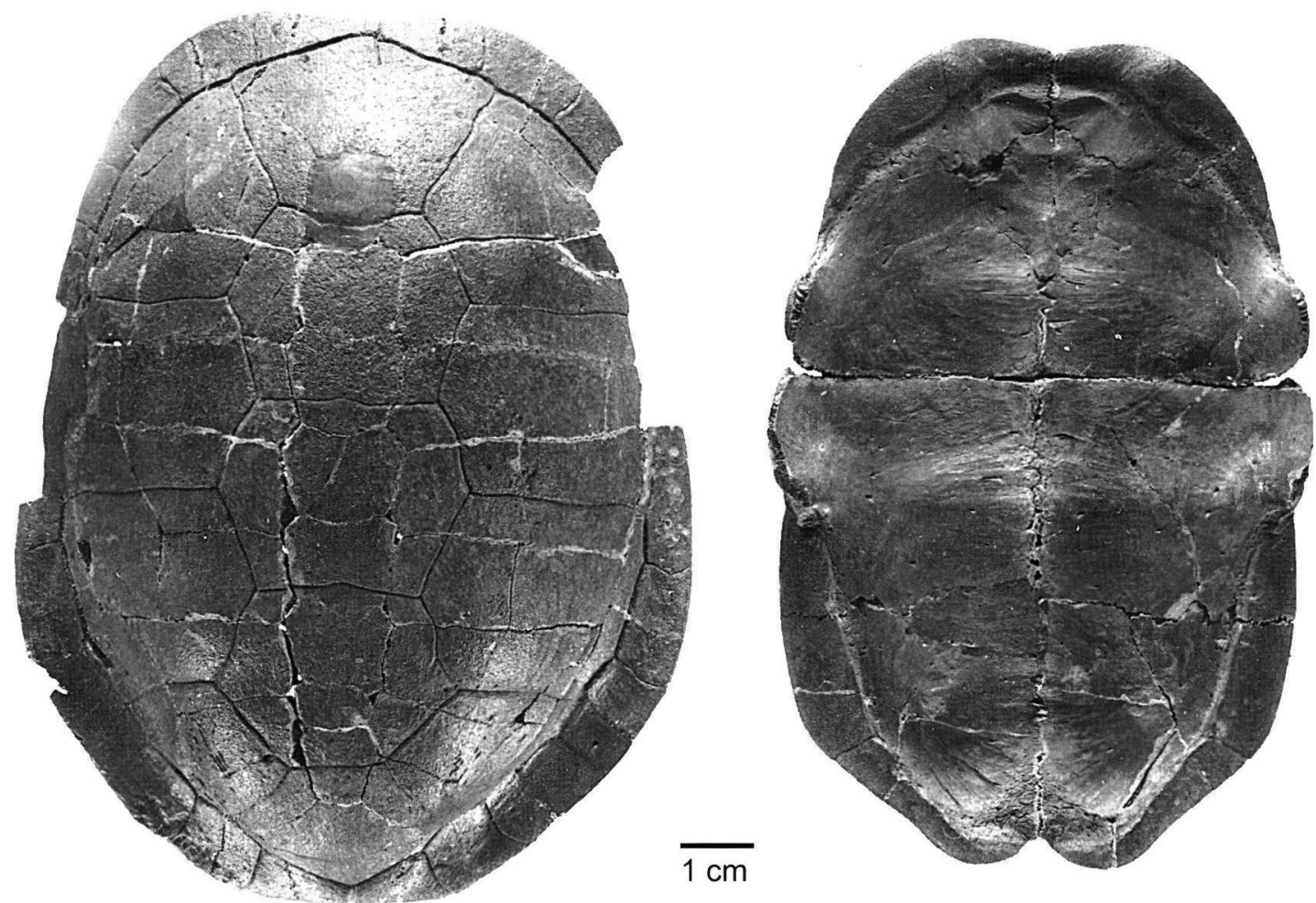

Abb. 2: Carapax und Plastron eines nahezu vollständigen Individuums der Europäischen Sumpfschildkröte (Emys orbicularis).

Fig. 2: Carapace and plastron of a nearly complete specimen of the European Pond Tortoise (Emys orbicularis).

Phalacrocorax carbo (Linnaeus, 1758) - Kormoran Material: 1 Sternum-Fragment, 2 Cervicalwirbel, 3 Lumbalwirbel, 1 Coracoid, 1 Pelvis

Die Funde stammen vermutlich von einem Individuum. Die Maße entsprechen rezentem Vergleichsmaterial. Heute lebt der Kormoran an Meeresküsten, Flüssen, Flussmündungen sowie an Seen. Seine Nahrung besteht vorwiegend aus Fisch (FERGUSONLees \& Willis 1983). Somit ist die Anwesenheit des Kormorans in Lehringen nicht ungewöhnlich, vermutlich nutzte er den See zum Fischfang.

\section{Ardea cinerea LinNAEus, 1758 - Graureiher}

Material: 1 Humerus, 1 Tibiotarsus, 1 Tarsometatarsus

Mehrere Fragmente von Ardea cinerea liegen vor. Die Stücke können anhand der von Kellner (1986) angegebenen morphologischen Merkmale eindeutig bestimmt und von der sehr ähnlichen Art $A$. purpurea abgegrenzt werden. In den Maßen liegen die Lehringer Stücke im Streubereich der rezenten Graureiher.

A. cinerea ist seit dem Oberpliozän bekannt und aus pleistozänen Fundstellen Deutschlands, Englands, Irlands, Frankreichs und Ungarns nachgewiesen (LAMBRECHT 1933).

Anzutreffen ist der Graureiher an Seen, Flïssen, Flussmündungen und Sümpfen (FERGUSON-LeEs \& Willis 1983). Das Nahrungsspektrum reicht von Fischen und Fröschen bis hin zu Mäusen, Schlangen und großen Insekten. Ebenso wie der Kormoran nutzte der Graureiher den Lehringer See vermutlich zum Fischfang.

Castor fiber Linnaeus, 1758 - Biber (Abb. 3)

Material: 1 Cranium, 4 craniale Fragmente, 3 isolierte Zähne, 1 Atlas, 1 Scapula, 1 Humerus, 1 Ulna, 3 Pelvis-Fragmente, 2 Femora, 1 Tibia

Es können mindestens drei Individuen identifiziert werden. Alle Tiere sind subadult, da die Epiphysen 

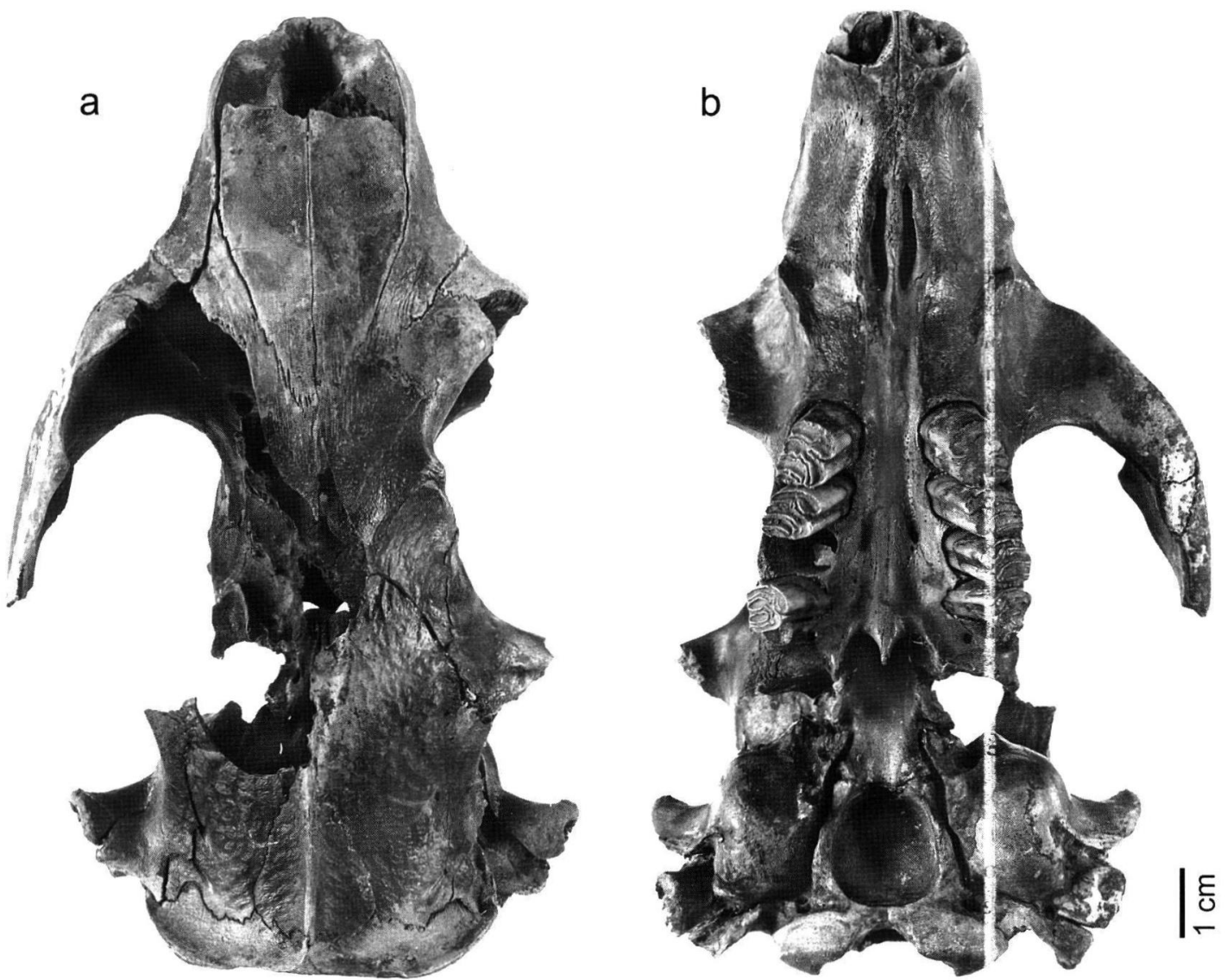

Abb. 3: Cranium eines Bibers (Castor fiber) aus Lehringen, a. dorsal, b. ventral.

Fig. 3: Cranium of beaver (Castor fiber) from Lehringen, a. dorsal, b. ventral.

noch nicht geschlossen sind. In den Maßen liegen die Stücke im Bereich rezenter und eemzeitlicher Biber. V. Reichenau (1912) gibt für den M3 des rezenten $C$. fiber eine länge von 5,0-7,1 mm und eine Breite von $6,0-7,1 \mathrm{~mm}$ an. Die vorliegenden Zähne messen in der Länge 6,9-7,0 $\mathrm{mm}$ und in der Breite 6,3-6,6 mm, womit sie im oberen Bereich der rezenten Biber liegen. Die jungpleistozänen Formen sind generell etwas größer.

C. fiber ist aus den eemzeitlichen Fundstellen der nördlichen Oberrheinebene sowie von Steinheim an der Murr (Baden-Württemberg), Burgtonna, WeimarStadtgebiet und Schönfeld belegt (v. KoENIGSWALD \& HeINRICH 1999).

Ökologisch bevorzugt $C$. fiber Gewässer mit holzreichen Ufern sowie Auenwälder. Er baut Burgen und Dämme aus gefällten Bäumen, wobei Bäume mit einem Stammdurchmesser is $20 \mathrm{~cm}$ bevorzugt werden. Ein aus Lehringen übe liefertes Holz zeigt die typischen 5-10 $\mathrm{mm}$ breiten : Ind gerippt erscheinenten Nagesn :r

Canis lupus Linnaeus, 1758 - Wolf

Material: 1 Tibia-Fragment

Canis lupus kann durch ein Tibia-Fragment nachgewiesen werden. Diese Tibia ist in der Mitte der Diaphyse gebrochen und nur der distale Teil liegt vor. Die geschlossene Epiphyse weist auf ein adultes Tier hin. Der Lehringer Wolf entspricht in seiner Größe dem rezenten europäischen Wolf und ist deutlich größer als C. l. mosbachensis.

C. lupus ist ein typisches Raubtier eemzeitlicher Faunen und wurde ebenfalls in den Schichten von Stuttgart-Untertürkheim (Baden-Württemberg), 
Neumark-Nord (Sachsen-Anhalt), Hunas/Hartmannshof (Bayern), Taubach und Weimar-Ehringsdorf (Thüringen) nachgewiesen (v. KoENIGSWALD \& HeinRICh 1999). Generell zeigt sich der Wolf als klimatisch indifferente Art und sein Habitat schließt außer den hochalpinen Zonen alle Lebensräume ein.

\section{Ursus cf. arctos - Braunbär}

Material: 1 Femur-Fragment

Der distale Teil des linken Femurs eines Ursiden ist überliefert. Das Stück ist kurz oberhalb der distalen Epiphyse abgebrochen und die Epiphyse ist vollständig verwachsen. Das Fragment ermöglicht lediglich eine Bestimmung auf Gattungsniveau. Die geringe Größe des Stückes deutet jedoch auf einen Braunbären hin. Im Gegensatz zu $U$. arctos ist $U$. spelaeus durchschnittlich etwa ein Drittel größer und wesentlich massiger gebaut. Auch Sickenberg (1969) weist diesen Fund vorbehaltlich $U$. arctos zu und begründet dies mit den ökologischen Gegebenheiten.

Im Eem ist der Braunbär aus den Fundstellen Stuttgart-Untertürkheim, Burgtonna, WeimarStadtgebiet, Taubach und der nördlichen Oberrheinebene bekannt (v. KoENiGSWALd \& HeinRICH 1999).
Felis silvestris SCHREBER, 1777 - Wildkatze

Material: 1 Metatarsale III

Ein bislang noch nicht beschriebenes Metatarsale III einer Felis silvestris liegt vor. Die Lehringer Wildkatze ist zwar noch nicht vollständig ausgewachsen, kann aber auf Grund von Vergleichsmaßen anderer eemzeitlicher Fundstellen sicher zu $F$. silvestris gestellt werden.

Funde dieser Kleinkatze sind relativ selten. Im Jungpleistozän ist $F$ silvestris aus Stuttgart-Untertürkheim, Burgtonna und Weimar-Stadtgebiet bekannt (v. Koenigswald \& Heinrich 1999).

Die rezente Wildkatze bevorzugt dichte Nadel-, Laub- und Mischwälder mit Lichtungen und Steinhalden. Eine Schneehöhe ab etwa $20 \mathrm{~cm}$ stellt den limitierenden Faktor des Habitats dar, da dann Probleme der Fortbewegung auftreten (Hemmer 1993).

Elephas antiquus (Falconer \& Cautley, 1847) Waldelefant (Abb. 4)

Material: 5 Molaren, isolierte Schmelzlamellen, 1 Cervicalwirbel, 7 Thoracalwirbel, 3 Wirbelfragmente, 2 Costae, 2 Scapulae, 1 Humerus-Fragment, 3 Femur-Fragmente, 1 Metacarpale, 6 Phalangen, diverse weitere Knochenfragmente

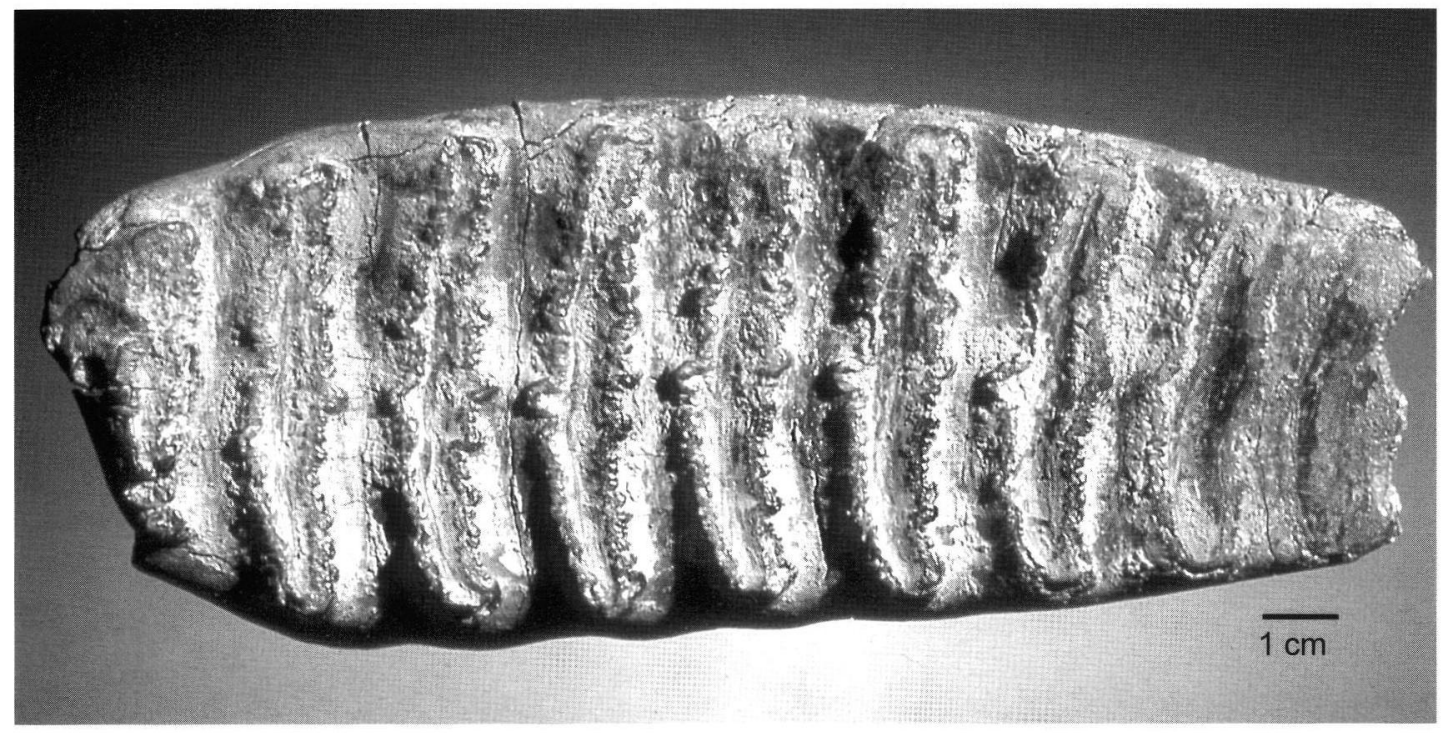

Abb. 4: Rechter m2 des Waldelefanten (Elephas antiquus).

Fig. 4: Right $\mathrm{m} 2$ of the Straight-Tusked Elephant (Elephas antiquus). 
In Lehringen wurde ein annähernd vollständiges, jedoch durch die Bergung stark beschädigtes Skelett eines Waldelefanten gefunden. Bereits bei der Entdeckung des Fundes zerstörte ein Bagger den Schädel weitgehend und während der Bergung gingen weitere Stücke verloren (DeIBEL-Rosenbrock 1960). ADAm (1951) bestimmt die Art und beschreibt einen etwa $2,50 \mathrm{~m}$ langen, geraden Stoßzahn, wie er für Waldelefanten typisch ist. Dieser Stoßzahn konnte allerdings nicht mehr aufgefunden werden. Eindeutige Merkmale für die Zuordnung des Skeletts zu Elephas antiquus liefert die signifikante Struktur der Molaren mit ihren weit auseinander stehenden Lamellen, die bei Mammuthus primigenius viel enger ausgebildet sind.

Der $\mathrm{m} 2$ befindet sich vollständig in Abrasion, und die ersten Lamellen des $m 3$ zeigen eine beginnende Abkauung. Der afrikanische Elefant (Loxodonta africana) zeigt dieses Stadium des Zahnwechsels im Alter von 35 bis 40 Jahren (Averianov 1996), weshalb der Lehringer Waldelefant vermutlich etwas jünger ist als die von ADAM (1951) geschätzten 45 Jahre.

Die Maße des Lehringer Individuums liegen im Bereich der Größenordnung der männlichen Waldelefanten aus Kiesäcker und Gröbern I. Bei Elefanten liegt generell ein starker Sexualdimorphismus vor, wobei weibliche Tiere kleiner sind. Der von ADAM (1951) beschriebene Stoßzahn weist anhand seiner Größe ebenfalls auf ein männliches Individuum hin. Nach einer Pollenanalyse an aus den Knochen entnommenen Sedimentresten stammt das Skelett des Waldelefanten aus dem Wärmemaximum des Eems (SicKenberg 1969). E. antiquus ist eine typische Form der Interglaziale und verschwindet jeweils mit dem Beginn der Glaziale. Er benötigte den klimatischen Bereich des Laubmischwaldes und bevorzugte ein stärker mediterran geprägtes Klima (v. KoENigswald 1988).

Der Lehringer Elefant erlangte an Berühmtheit, da im Verband mehrere Feuersteinartefakte sowie eine Eibenholzlanze gefunden wurden. Der Entdecker der Stoßlanze, Отто Vogt, berichtet, dass er diese im Bereich der Rippen gefunden habe (Thieme \& VeIL 1985). Es liegt jedoch über die Fundsituation keine genaue Beschreibung oder Zeichnung vor. ADAM (1951) interpretiert auf Grund der Fundumstände eine Elefantenjagd, JАСОв-FriESEN (1956) und Thieme \& Veil (1985) bekräftigen dies. V. KoE-
NIGSWALD (2002) nimmt an, dass die vermutlichen Jäger das Tier bereits verstorben vorfanden und mit der Lanze lediglich den Tod überprüften.

\section{Equus sp. - Pferd}

Material: 2 isolierte obere Molaren, 2 craniale Fragmente, 1 Radius mit Ulna, 2 Femora

Nur wenige Fragmente eines subadulten Pferdes liegen vor. Vermutlich stammen die Funde aufgrund der Größe und des Ossifikationsstadiums von einem Individuum. Die isolierten Zähne sind typisch caballoid ausgebildet. Eine genaue Artbestimmung kann anhand des überlieferten Materials nicht durchgeführt werden.

Rezente Pferde sind vor allem in Steppenlandschaften verbreitet, doch im Pleistozän treten sie ebenso in Waldlandschaften und Tundren auf. Bekannt ist die Gattung Equus aus vielen eemzeitlichen Fundstellen, wie der nördlichen Oberrheinebene, Steinheim an der Murr, Burgtonna, Weimar-Stadtgebiet und Taubach (v. Koenigswald \& Heinrich 1999). Die Pferde gehören zu den klimaindifferenten Arten und treten in glazialen wie interglazialen Fundstellen auf.

\section{Equus cf. hydruntinus - Wildesel}

Material: 1 Scapula, 1 Femur, 1 Tibia

Interessant sind die Funde eines weiteren, wesentlich kleineren, adulten Equiden. Die geringen Größe lässt vermutet, dass es sich hierbei um Equus hydruntinus handelt.

E. hydruntinus tritt häufig unter warmzeitlichen Bedingungen, aber auch in kälteren bis kaltzeitlichen Faunen auf (ForSTEN \& ZiegLer 1995). In den Faunen ist der Wildesel meist nur durch wenige Funde dokumentiert; sein Fehlen kann jedoch auch auf Fundlücken zurückgeführt werden. Daher kann durch sein Auftreten keine eindeutige Klimaaussage getroffen werden (FORSTEN \& Z ZIEGLER 1995).

Aus Deutschland ist E. hydruntinus aus den jungpleistozänen Fundstellen Stuttgart-Untertürkheim und Weimar-Stadtgebiet bekannt (v. KoENIGSWALD \& HeINRICH 1999).

Stephanorhinus hemitoechus (FALCONER, 1868) Steppennashorn (Abb. 5)

Material: $1 \mathrm{M} 3$

Von einem Nashorn ist aus Lehringen nur ein isolierter Zahn bekannt. Die Schmelzoberfäche ist 


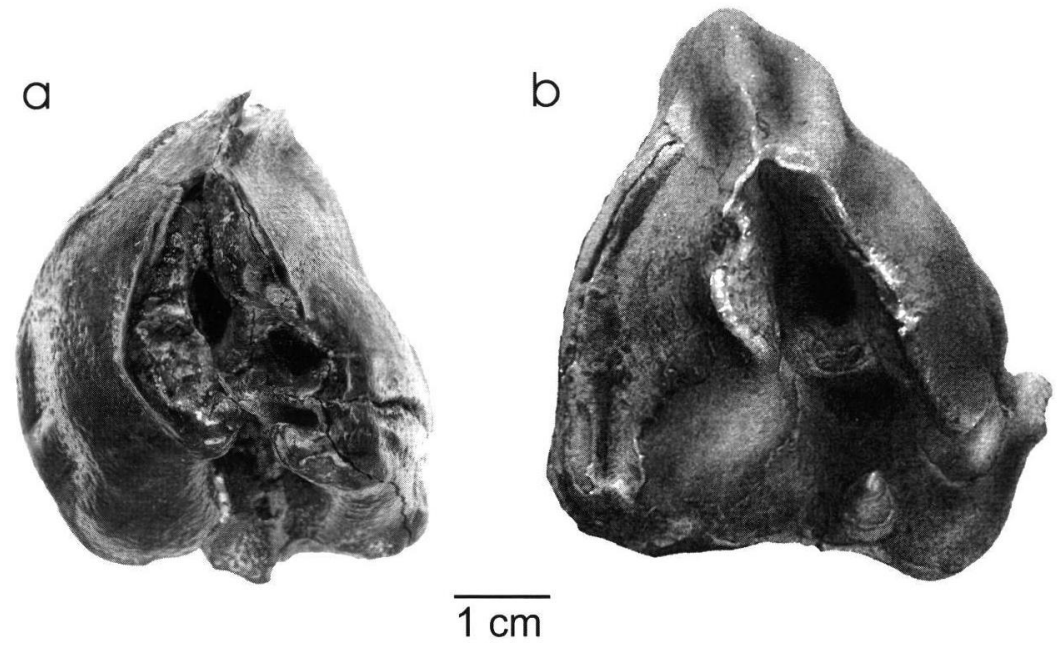

Abb. 5: Gegenüberstellung eines M3 von Stephanorhinus hemitoechus.

a. aus Lehringen b. aus dem Heppenloch bei Gutenberg (Baden-Württemberg) (STAESCHE 1941)

Fig. 5: Comparision of the M3 of Stephanorhinus hemitoechus.

a. from Lehringen b. from Heppenloch near Gutenberg (Baden-Württemberg) (STAESCHE 1941)

rau und im Mediantal sind noch Reste von Zahnzement erhalten. Diese beiden Merkmale sprechen nach STAEsCHE (1941) für Stephanorhinus hemitoechus, da bei Stephanorhinus kirchbergensis die Schmelzoberfläche wesentlich glatter ist und nur selten Zahnzement angelegt wird. Ein weiteres Kriterium stellt die geringere Größe des Zahnes gegenüber $S$. kirchbergensis dar. Die dritten Molaren von $S$. hemitoechus sind in der Tendenz kleiner als die von $S$. kirchbergensis. Die Zuordnung SicKENBERGs (1969) zu S. kirchbergensis kann daher nicht bestätigt werden. Das aus der nördlichen Oberrheinebene bekannte sehr kleine Nashorn, das v. Koenigswald (1988) als Dicerorhinus cf. megarhinus aufführt, liegt in den Maßen im Bereich des Lehringer Stückes, kann aber nach morphologischen Kriterien ausgeschlossen werden.

Aus dem Eem ist $S$. hemitoechus aus den Fundstätten der nördlichen Oberrheinebene, StuttgartUntertürkheim, Burgtonna, Weimar-Stadtgebiet und Taubach belegt (v. Koenigswald \& Heinrich 1999).

Ökologisch zeigen sich an der Skelettmorphologie Anpassungen an offene Habitate. Auf Grund der hochkronigen Zähne und der tiefen Kopfhaltung kann auf Grasnahrung geschlossen werden; jedoch deutet die ektolophodonte Morphologie der Kau- fläche auf ein intermediäres Nahrungspektrum tief wachsender Vegetation hin (ForTELIUs 1982).

\section{Megaloceros giganteus (BLUMENBACH, 1803) - Riesenhirsch}

Material: 5 Thoracalwirbel, 6 Lumbalwirbel, 1 Sacralwirbel, diverse Costae, 2 Scapulae, 2 RadiusFragmente, 3 Pelvis-Fragmente

Im Vergleich zum Material von Cervus elaphus und Dama dama aus Lehringen sind von Megaloceros giganteus nur wenige Stücke überliefert.

Ein Wirbelsäulenabschnitt vom zehnten Thoracalwirbel bis zum ersten Sacralwirbel liegt vor. Die identifizierten Merkmale am postcranialen Material sichern die taxonomische Zuordnung. Durch eine Pollenanalyse ist bekannt, dass die Stücke aus dem Wärmemaximum stammen (SICKENBERG 1969).

Im Eem ist der Riesenhirsch weit verbreitet; neben Lehringen tritt er auch in der nördlichen Oberrheinebene, in Stuttgart-Untertürkheim, in Neddenaverbergen (Niedersachsen), in Burgtonna, in Weimar-Stadtgebiet und in Taubach auf (v. KoENigSWALd \& HeINRICH 1999). Außerhalb von Deutschland ist er im Eem und Weichsel von Großbritannien, Italien, Ungarn, der Slowakischen Republik und Polen nachgewiesen (LISTER 1994). M. giganteus ist eine klimatisch indifferente Art. 
Der Riesenhirsch tritt in mediterranen bis subarktischen Klimazonen auf, nur unter arktischen Verhältnissen ist er nicht mehr anzutreffen. Ökologisch ist $M$. giganteus in offenen Landschaften als auch in stark bewaldeten Gebieten verbreitet und zeigt eine große Anpassungsfähigkeit an unterschiedliche Biotope.

\section{Cervus elaphus Linnaeus, 1758 - Rothirsch}

Material: 4 Maxillar-Fragmente, 4 MandibularFragmente, 1 schädelechtes Geweih, 1 Atlas, 2 Cervicalwirbel, 1 Thoracalwirbel, diverse Costae, 1 Scapula, 3 Humeri, 1 Radius mit Ulna, 4 Carpalia, 1 Metacarpus, 1 Pelvis, 1 Femur, 1 Tibia, 1 Calcaneus

Mindestens fünf Individuen vom Cervus elaphus sind überliefert. Dabei handelt es sich um einen sehr starken, etwa 15 Jahre alten Hirsch, zwei etwa 10 Jahre alte Hirsche, einen etwa 4-5 Jahre alten Hirsch und eine adulte Hirschkuh. Es liegt ein artikuliertes Vorderbein von der Scapula bis zum Metacarpus (V $5030 \mathrm{a}-\mathrm{g}$ ) eines adulten Tieres vor. Die Rothirsche aus Lehringen sind sehr kräftig und liegen nach den Maßen im oberen Streubereich anderer jungpleistozäner Funde. C. elaphus gehört zu den klimaindifferenten Arten.

\section{Dama dama FRISCH, 1775 - Damhirsch}

Material: 1 schädelechtes Geweih, 4 craniale Fragmente, 7 Cervicalwirbel, 5 Thoracalwirbel, 5 Lumbalwirbel, diverse Costae, 2 Humeri, 1 Radius mit Ulna, 2 Metacarpi

Aus Lehringen liegen drei Individuen der Art Dama dama vor. Es handelt sich um einen ca. 2-3 jährigen und einen ca. 5-6 jährigen Hirsch sowie um eine adulte Damkuh. Das Hinterhaupt V 5100.1 zeigt eine stark s-förmig verlaufende Linea nucha, wohingegen am Stück V 5100.2 diese nur schwach s-förmig ausgebildet ist. Rezente Damhirsche haben diese geschwungene Linea nucha nicht mehr, die Hirsche aus Neumark-Nord dagegen zeigen diese immer. Damit bilden die Lehringer Hirsche eine Mittelstellung zwischen $D$. dama geiselana und $D$. dama dama (vgl. Pfeiffer 1997).

Im Eem erreicht der Damhirsch unter optimalen warmzeitlichen Bedingungen seine größte Verbreitung. In Norddeutschland wurde $D$. dama neben Lehringen auch in Deutsch-Evern (Niedersachsen) nachgewiesen (STOLLER 1918). Weitere eemzeit- liche Funde stammen aus Burgtonna, WeimarEhringsdorf, Taubach und der nördlichen Oberrheinebene. Außerhalb Deutschlands ist D. dama auch in England, den Niederlanden, Frankreich und im gesamten Mittelmeerraum nachgewiesen (Pfeiffer 1999). Mit dem Einsetzen des WeichselGlazials verschwindet er in den nördlichen Arealen und hält sich nur noch im Mittelmeerraum (HALTENORTH 1959).

\section{Capreolus capreolus (LinnaEus, 1758) - Reh}

Material: 1 Maxillarfragment, 1 Scapula, 1 Tibia, 1 Metacarpus

Das Material aus Lehringen liegt im Streubereich der rezenten Rehe und ist deutlich kleiner als $C$. pygargus und $C$. suessenbornensis. Es liegen mindestens zwei Individuen vor, da das Maxillarfragment in den Proportionen deutlich größer ist als das postcraniale Material. Da bei Cerviden der Sexualdimorphismus stark ausgeprägt ist, handelt es sich bei dem vorliegenden postcranialen Material vermutlich um ein weibliches Tier.

Aus dem Eem Deutschlands sind neben Lehringen noch weitere Funde von C. capreolus aus StuttgartUntertürkeim, der nördlichen Oberrheinebene, Burgtonna, Weimar-Stadtgebiet, Taubach und Schönfeld bekannt (v. Koenigswald \& Heinrich 1999). Ebenso wie die Cerviden wandert Capreolus aus Zentral- und Ostasien nach Europa ein. Im letzten Interglazial erreicht $C$. capreolus in Eurasien eine maximale Verbreitung. In Europa ist das Vorkommen des Rehs auf Interglaziale beschränkt. Begrenzender Faktor der Verbreitung von C. capreolus sowie C. pygargus ist die Schneehöhe. Erreicht diese mehr als $50 \mathrm{~cm}$, werden Fortbewegung und Nahrungsaufnahme soweit erschwert, dass eine dauerhafte Besiedlung nicht möglich ist (PFEIFFER 1998).

\section{Bos primigenius (Bojanus, 1827) - Ur oder Auer-} ochse

Material: diverse craniale Fragmente, isolierte Oberkieferzähne (P2-M3), 3 Unterkieferfragmente, 2 Zungenbeinfragmente, 1 Atlas, 1 Axis, 8 weitere Cervicalwirbel, 21 Thoracalwirbel, 11 Lumbalwirbel, 4 Sacralwirbelfragmente, 5 Scapulae, 4 Humeri, 3 Radii mit Ulnae, 1 Radius, 1 Ulna, 3 Metacarpalia, 6 Pelvis-Fragmente, 2 Femora, 4 Femur-Fragmente, 2 Tibiae, 1 Tibia-Fragment, 
2 Metatarsalia, 3 Astragali, 3 Calcanei, 3 Patellae, 15 Phalangen, 14 Carpalia und Tarsalia, diverse Costae

Die Funde deuten auf mindestens drei Individuen hin. Dabei ist ein Tier annähernd vollständig, ein zweites etwa zur Hälfte und ein drittes Tier nur durch wenige Einzelfunde überliefert. Nach dem Epiphysenschluss und dem Abkauungsgrad der erhaltenen Zähne zu urteilen, sind die Tiere ca. 3-4 Jahre alt (HABERMEHL 1975). Die morphologischen Merkmale, vor allem an den Langknochen, führen zu einer sicheren taxonomischen Zuordnung.

$B$. primigenius ist ein typisches Element warmzeitlicher Faunen, dabei zeigt er jedoch große klimatische Toleranzen und ist nicht nur auf das Optimum der Interglaziale beschränkt, sondern tritt auch in kaltzeitlichen Biotopen auf (v. KOENIGSWALD 1999a). In Kaltzeiten mit starkem maritimen Einfluss und somit größerer Feuchtigkeit und milden Wintern ist eine Besiedlung durch $B$. primigenius möglich. Nimmt dagegen das kontinentale Klima zu, verschwindet der Auerochse (v. KoENIGSWALD 1999a).

\section{Die Rekonstruktion des Klimas auf Grund der Fauna}

Die eemzeitliche Fauna von Lehringen zeigt einen einheitlichen, warmzeitlichen Charakter, der sich vor allem im Auftreten thermophiler Elemente wie Silurus glanis, Emys orbicularis, Elephas antiquus, Stephanorhinus hemitoechus, Dama dama und Capreolus capreolus äußert. Diese Tiere sind ausschließlich aus Interglazialen bekannt. Die übrigen in Lehringen nachgewiesenen Formen sind klimaindifferent; reine kaltzeitliche Formen treten nicht auf.

Silurus glanis weist auf warme Sommer hin, da der Laichvorgang erst bei einer Wassertemperatur von $18-20^{\circ} \mathrm{C}$ einsetzt (BöHME 1996). Er tritt auch heute noch in Flüssen und Seen Mitteleuropas auf. Die enorme Größe der Fische weist ebenfalls auf optimale ökologische und klimatische Bedingungen hin. Auch bei der Sumpfschildkröte Emys orbicularis handelt es sich um einen wichtigen Klimaindikator. Sie ist heute die am weitesten nach Norden vorgedrungene Schildkröte und tritt auch rezent in Deutschland noch in wenigen Reliktarealen auf (Gläss \& Meusel 1994). Im Eem war sie jedoch wesentlich weiter verbreitet und ist in zahlreichen interglazialen Fundkomplexen nachgewiesen (UlLRICH 1978). Den wesentlichen limitierenden Faktor der Verbreitung von E. orbicularis stellen die sommerlichen Temperaturen dar (GLäss \& Meusel 1994). Die Eier benötigen über einen längeren Zeitraum mindestens $24^{\circ} \mathrm{C}$ zur Entwicklung. Bei den rezenten Formen schlüpfen die Jungen nach 50-80 Tagen, in kalten Sommern sogar erst im folgenden Frühjahr. In Lehringen erreichen die Schildkröten nicht ihre maximale Größe. Dies kann auf das Individualalter zurückgeführt werden; andererseits kann sich hierin auch eine klimatische Anpassung zeigen.

In der Säugetierfauna treten Damhirsch und Waldelefant auf. Auch Bos primigenius tritt meist in Interglazialen auf, wandert aber bereits bei zunehmender Wiedererwärmung ein (v. KoENIGSWALD 1999a). Das Reh ist eine weitere Leitform der Interglaziale und aus fast allen eemzeitlichen Fundstellen bekannt. Pfeiffer (1998) betont, dass seine Verbreitung durch die Schneehöhe eingeschränkt ist. Beträgt diese über $50 \mathrm{~cm}$, so entstehen für Capreolus capreolus wie auch für Capreolus pygargus Lokomotionsschwierigkeiten. Es ist jedoch schwierig auf Mindesttemperaturen zu schließen.

In der Schichtenfolge zeigt sich anhand des Pollenprofils eine stetige Erwärmung bis zum Klimamaximum, dann eine kontinuierliche Abkühlung (SELLE 1962). Das nur 11.000 Jahre andauernde Eem ist mit mittlerweile über 200 untersuchten Lokalitäten zwischen den Niederlanden und Weißrussland sicherlich das am besten paläobotanisch erforschte Interglazial (LiтT 2001). LiтT (1994) zeigt eine erstaunliche Übereinstimmung der Waldentwicklung innerhalb dieser Gebiete und postuliert eine durchgängige Bewaldung während des gesamten EemZyklusses in Mitteleuropa. Dabei kann die JuliTemperatur des Eem gegenüber dem Holozän höchstens $2-3^{\circ} \mathrm{C}$ höher gewesen sein (FRENZEL 1989). Das Auftreten von Silurus glanis und Emys orbicularis zeigt, dass die durchschnittlichen Sommertemperaturen sicherlich über dem Grenzwert von $20^{\circ} \mathrm{C}$ gelegen haben. Im Winter kann durchaus Frost geherrscht haben, so dass der See zeitweise zufror. Dies beeinträchtigt nicht die an den aquatischen Lebensraum angepassten Taxa Emys orbicularis und Castor fiber (vgl. Colditz 1994, HallerProbst 1997). 


\section{4 Ökologie}

Die fossile Wirbeltierfauna ermöglicht eine differenzierte palökologische Aussage über Gewässer und Umland des Fundkomplexes von Lehringen. In der nachgewiesenen Gemeinschaft schließt keine Art ein anderes Taxon ökologisch grundsätzlich aus. Alle 18 aus Lehringen bekannten Taxa sind auch aus Fundstellen des jüngeren Pleistozäns Mitteleuropas belegt (v. KoENigSWALd \& HeinRICH 1999). In Tabelle 2 wird ein Vergleich zu anderen zeitgleichen Fundstellen gegeben. Die Lehringer Fauna spiegelt das typische eemzeitliche Faunenbild Mitteleuropas wider.

Die meisten Arten treten noch heute im weiteren Umland der Fundstelle auf. Ein Faunenvergleich durch RaufuB (2001) ergibt, dass ein rezentes Vergleichsareal im Gebiet der Fundstelle zu finden ist. Daher kann bei der Analyse auf aktualistische Beobachtungen zurückgegriffen werden. Die Tiere, die heute nicht mehr auftreten, sind entweder ausgestorben (Elephas antiquus, Stephanorhinus hemitoechus, Megaloceros giganteus und Bos primigenius) oder wurden durch den Menschen im Gebiet ausgerottet (Ursus arctos, Canis lupus und Felis silvestris). Das Aussterben von $E$. antiquus und S. hemitoechus wird auf eine umfassende ökologische Veränderung während des letzten Glazials im Kerngebiet zurückgeführt (v. KoENIGSWALD 1999b). Generell wandern kaltzeitliche Elemente aus den Kerngebieten Osteuropas mit einsetzender Abkühlung nach Europa ein. Währenddessen sterben die

Tab. 2: Artbestand aus Lehringen im Vergleich zu anderen eemzeitlichen Fundstellen Deutschlands (nach v. KoenigsWALD \& HEINRICH 1999).

Tab. 2: The vertebrate fauna of Lehringen in comparision with other Eemian localities in Germany (after v. KoENigsWALD \& HeINRICH 1999).

\begin{tabular}{|c|c|c|c|c|c|c|c|c|c|c|c|}
\hline & 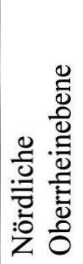 & 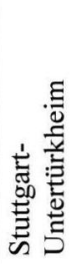 & 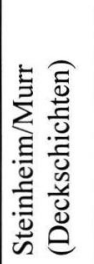 & 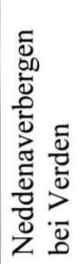 & 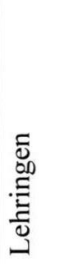 & 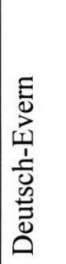 & 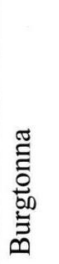 & 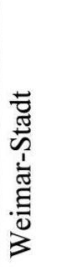 & 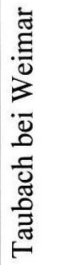 & 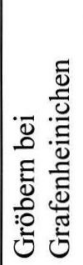 & 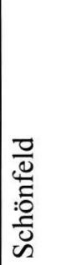 \\
\hline Esox lucius & & & $\bar{x}$ & $\mathrm{x}$ & $\mathrm{x}$ & & & & & $\bar{x}$ & $\bar{x}$ \\
\hline Silurus glanis & & & & & $\mathrm{x}$ & & & & & & $\mathrm{x}$ \\
\hline Emys orbicularis & & & & $\mathrm{x}$ & $\mathrm{x}$ & & $\mathrm{x}$ & $\mathrm{x}$ & $\mathrm{x}$ & & $\mathrm{x}$ \\
\hline Phalacrocorax carbo & & & & & $\mathrm{x}$ & & & & & & \\
\hline Ardea cinerea & & & & & $\mathrm{x}$ & & & & & & \\
\hline Castor fiber & $\mathrm{x}$ & & $\mathrm{x}$ & $\mathrm{x}$ & $\mathrm{x}$ & & $\mathrm{x}$ & $\mathrm{x}$ & $\mathrm{x}$ & & $\mathrm{x}$ \\
\hline Canis lupus & & $\mathrm{x}$ & & & $\mathrm{x}$ & & & & $\mathrm{x}$ & & $\mathrm{x}$ \\
\hline Ursus arctos & & $\mathrm{x}$ & & & $?$ & & $\mathrm{x}$ & $\mathrm{x}$ & $\mathrm{x}$ & & \\
\hline Felis silvestris & & $\mathrm{x}$ & & & $\mathrm{x}$ & & $\mathrm{x}$ & $\mathrm{x}$ & & & \\
\hline Elephas antiquus & $\mathrm{x}$ & $\mathrm{x}$ & & & $\mathrm{x}$ & & $\mathrm{x}$ & $\mathrm{x}$ & $\mathrm{x}$ & $\mathrm{x}$ & \\
\hline Equus sp. & & & $x$ & & $\mathrm{x}$ & & $\mathrm{x}$ & $x$ & $\mathrm{x}$ & & \\
\hline Equus hydruntinus & & $\mathrm{x}$ & & & $?$ & & $\mathrm{x}$ & $\mathrm{x}$ & & & \\
\hline Stephanorhinus hemitoechus & $\mathrm{x}$ & $\mathrm{x}$ & & & $\mathrm{x}$ & & $\mathrm{x}$ & $\mathrm{x}$ & $\mathrm{x}$ & & \\
\hline $\mid$ Megaloceros giganteus & $\mathrm{x}$ & $\mathrm{x}$ & & $\mathrm{x}$ & $\mathrm{x}$ & & $\mathrm{x}$ & $\mathrm{x}$ & $\mathrm{x}$ & & \\
\hline Cervus elaphus & $\mathrm{x}$ & $\mathrm{x}$ & & $\mathrm{x}$ & $\mathrm{x}$ & $\mathrm{x}$ & $\mathrm{x}$ & $\mathrm{x}$ & $\mathrm{x}$ & $\mathrm{x}$ & $\mathrm{x}$ \\
\hline Dama dama & $\mathrm{x}$ & & & $\mathrm{x}$ & $\mathrm{x}$ & $\mathrm{x}$ & $\mathrm{x}$ & $\mathrm{x}$ & $\mathrm{x}$ & $\mathrm{x}$ & \\
\hline Capreolus capreolus & $\mathrm{x}$ & $\mathrm{x}$ & & & $\mathrm{x}$ & & $\mathrm{x}$ & $\mathrm{x}$ & $\mathrm{x}$ & & $\mathrm{x}$ \\
\hline Bos primigenius & $\mathrm{x}$ & $\mathrm{x}$ & & & $\mathrm{x}$ & & & & & & \\
\hline
\end{tabular}


warmzeitlichen Elemente im temporären Verbreitungsgebiet aus, wc jei deren Kerngebiet vermutlich im Mittelmeerraum liegt. Nach v. KoENIGSWALD (1999b) erreichte die Klimaverschlechterung der letzten Eiszeit erstmals die Kerngebiete der interglazialen Fauna. Incolgedessen starben einige Tiere aus. Die Damhirche hielten sich im SE des Kerngebietes, wanćerten im Holozän jedoch nicht mehr nach Mitteleuropa ein (v. Koenigswald 1991). Erst durch (ien Menschen wurden sie in der frühen Neuzeit wieder in Mitteleuropa angesiedelt. Über die Ökologit des Sees geben vor allem die vorkommenden Fischarten Esox lucius und Silurus glanis Aufschluss, cia sie eine autochthone Einbettung voraussetzen. Der Hecht lebt heute vorwiegend in ruhigan Gewässern und Seen und bevorzugt üppig be:vachsene Stellen, an denen er als Standjäger seiner Beute auflauern kann (MüLlER 1987, Vilcinskas i993). Der submerse Pflanzenbewuchs kann eberifalls anhand der fossilen Pflanzenreste bestätigt werden. Der Wels bevorzugt schlammigen Grun 1 und weniger dichten Pflanzenbewuchs (MıнÁLIK 1995). Die enorme Größe beider Fischarten lässt auf ein nährstoffreiches Gewässer schließen.

Die Sumpfschildkröte bevorzugt wie der Wels trübschlammige Gewä:ser (GLäss \& Meusel 1994). Die Thermoregulation bei Schildkröten erfolgt vorwiegend verhaltensgesteuert, deshalb benötigen sie freie Stellen zum Scnnen und meiden Gewässer mit dicht bewachsene, Uferzonen (HaLler-Probst 1997).

Unter den in Lehringen nachgewiesenen Wirbeltieren steller die Säugetiere die artenreichste Gruppe dar und geben weitere wichtige Informationen über das Unland des Sees. Es treten vorwiegend waldbewo inende Tiere (Elephas antiquus, Ursus arctos, Felis silvestris, Megaloceros giganteus, Cervus elaphus, Dima dama, Capreolus capreolus und Bos primigeniu.) auf. Weiterhin treten die steppenbetonten Tiere Equus sp., Equus cf. hydruntinus, Stephanorhinus hen itoechus und Megaloceros giganteus auf, obwohl in den Pollenprofilen eine durchgängige Bewaldung dokumentiert ist. Auch aus anderen eemzeitlichen Fundstellen wie Taubach bei Weimar, Burgtonna, Weimar-Stadt, der nördlichen Oberrheinebene und Stuttgart-Untertürkheim sind aus dem Fossilbericht wald- und steppenbetonte Formen nebeneinander belegt. Da- her sollte überdacht werden, ob die typischen Steppenanzeiger wirklich eine solche Aussagekraft besitzen.

In dieser Interglazialfauna fehlen jedoch die Exoten wie Hippopotamus und Bubalus. Diese Gattungen sind aus den Fundstellen der nördlichen Oberrheinebene bekannt. Auch in England ist Hippopotamus aus dem Ipwichian II belegt (STUART 1976). Jedoch reicht das Verbreitungsgebiet dieser Arten nicht weiter nach Osten. Die Verbreitung des Waldelefanten dünnt zwar nach Osten aus, reicht aber bis nach Warschau und damit sehr viel weiter als das Areal von Hippopotamus (JAKUBOwSKI et al. 1968).

Das Auftreten von Wasserbüffel und Flusspferd setzt einen stark atlantischen Klimaeinfluss voraus. Das Fehlen dieser Arten im gesamten nördlichen Raum kann auf die regionale Klimadifferenzierung für Teilabschnitte des Eems zurückgeführt werden.

\section{Zusammenfassung}

Aus den anhand von Pollenanalysen ins Eem gestellten Mergelschichten der Fundstelle Lehringen bei Verden (Aller) sind 18 Wirbeltiertaxa belegt. Im Artenspektrum zeigt sich eine typische eemzeitliche Fauna Mitteleuropas mit den Taxa: Esox lucius, Silurus glanis, Emys orbicularis, Phalacrocorax carbo, Ardea cinerea, Castor fiber, Ursus cf. arctos, Canis lupus, Felis silvestris, Elephas antiquus, Equus sp., Equus cf. hydruntinus, Stephanorhinus hemitoechus, Megaloceros giganteus, Cervus elaphus, Dama dama, Capreolus capreolus, Bos primigenius.

Die Funde der Osteichthyes, Reptilia und Aves wurden erstmals bearbeitet. Die von SiCKENBERG (1969) beschriebene Mammaliafauna wurde revidiert, dabei zeigen sich Abweichungen im Materialbestand. Die Zusammensetzung der Fauna deutet auf einen bewaldeten Lebensraum, in dem auch eher steppenbetonte Tiere wie Equus sp., Equus hydruntinus und Stephanorhinus hemitoechus auftreten. Die auftretenden Taxa schließen sich ökologisch nicht aus und sind ebenfalls in anderen eemzeitlichen Faunenassoziationen belegt. Daher gilt es, die sogenannten Steppenindikatoren auf ihre Aussagekraft hin zu überprüfen.

In der Faunenzusammensetzung sind die Cerviden am stärksten vertreten. Über die Population kann auf Grund der geringen Individuenzahl keine Aus- 
sage getroffen werden. Pflanzenfresser dominieren generell in Faunengemeinschaften, während Carnivoren wie auch in Lehringen nur in geringer Anzahl auftreten.

Die Funde in Lehringen spiegeln eine natürliche Sterberate innerhalb des Sedimentationszeitraums wider. Aus der rezenten Fauna ist bekannt, dass verletzte und kranke Tiere häufig zur Kühlung ins Wasser gehen. Dies trifft vielleicht für den Waldelefanten zu, der in situ im Verband mit menschlichen Artefakten gefunden wurde (ADAM 1951).

Die aquatischen bzw. subaquatisch angepassten Arten weisen auf einen ruhigen See mit einem schlammigen Grund und dichtem bis weniger dichtem Pflanzenbewuchs hin. Anhand des Pollenspektrums zeigt sich eine Erwärmung bis zum Klimamaximum mit einer sich anschließenden, kontinuierlichen Abkühlung zum Glazial. Im Klimamaximum können die Juli-Temperaturen etwa $2-3^{\circ} \mathrm{C}$ höher gewesen sein als im Holozän (Frenzel 1989). Ein Vergleichsareal ähnlicher Faunengemeinschaften liegt auch rezent im Gebiet der Fundstelle (RAufub 2001).

Exotische Faunenbestandteile wie Hippopotamus amphibius und Bubalus murrensis fehlen in der Lehringer Fauna. Diese Arten fehlen im gesamten nördlichen Raum, was auf regionale Klimadifferenzierung zurückgeführt werden kann.

\section{Danksagung}

Danken möchte ich Herrn Prof. Dr. W. v. KoenigsWALD, Institut für Paläontologie der Universität Bonn, für die Themenstellung und Betreuung meiner Diplomarbeit, die die Basis dieser Publikation bildet. Dem Historischen Museum Verden unter der Leitung von Herrn Dr. B. Emingholz danke ich für die Bereitstellung des Skelettmaterials. Bedanken möchte ich mich ferner bei Frau Dr. T. Pfeiffer für zahlreiche anregende Diskussionen. Ich danke weiterhin Herrn G. OlesChInSKI, Institut für Paläontologie der Universität Bonn, für die ausgezeichneten Fotos und Frau D. Kranz, Institut für Paläontologie der Universität Bonn, für die hilfreiche Unterstützung bei der graphischen Darstellung.

\section{Literatur}

ADAM, K.D. (1951): Der Waldelefant von Lehringen, eine Jagdbeute des diluvialen Menschen. - Quartär, 5: 79-92, Bonn.

Averianov. A.O. (1996): Sexual dimorphism in the mammoth skull, teeth and long bones. - In: Shoshani, J. \& Tassy, P. [eds.]: The Proboscidea, 203-213, Oxford Univ. Press., N.Y.

Boettger, C.R. (1954): Die Molluskenfauna des Interglazials von Lehringen bei Verden in Niedersachsen. - N. Jb. Geol. Paläont., Abh., 100/2: 247-285, 16 Abb., 2 Kt., 2 Tab., Stuttgart.

Böнme, G. (1996): Fischreste aus dem Eem-Interglazial von Schönfeld bei Calau, Niederlausitz. - Natur u. Landsch. Niederlausitz, Sonderheft, 2: 9-48, Cottbus.

- (1997): Fossile Fischfauna aus dem Känozoikum Deutschlands. - Quartär, 47/48: 113-138, Bonn.

Colditz, G. (1994): Der Biber. - Forum Artenschutz, 64 S., Augsburg.

Deibel-Rosenbrock, W. (1960): Die Funde von Lehringen. - Stader Jb. 1960: 65 S., Stade.

Ferguson-Lees, J. \& Willis, I. (1983): Vögel Mitteleuropas. - 2. Aufl., 352 S., 2130 Abb., 285 Kt., München (BLV).

Forsten, A. \& Ziegler, R. (1995): The horses (Mammalia, Equidae) from the early Wuermian of Villa Seckendorff, Suttgart-Bad Cannstatt, Germany. Stuttgarter Beitr. Naturkd., B, 224: 22 pp., 1 pl., 10 figs, 6 tabs., Stuttgart.

Fortelius, M. (1982): Ecological aspects of dental functional morphology in the Plio-Pleistocene Rhinoceroses of Europe. - In: KURTÉN, B. (ed.): Teeth: form, function and evolution. - Columbia Univ. Press, 168-181, New York.

Frenzel, B. (1989): Projektgruppe “Terrestrische Paläoklimatologie" im Rahmen des nationalen Klimaforschungsprogramms der Regierung. - Akad. Wiss. Lit. Mainz 1949-1989: 453-461, Stuttgart (Steiner).

Gläss, H. \& Meusel, W. (1994): Die Süßwasserschildkröten Europas. - N. Brehm-Bücherei., 418: 77 S., 48 Abb., Magdeburg (Westarp Wissenschaften).

Habermehl, K.-H. (1975): Altersbestimmungen bei Haustieren, Pelztieren und beim jagdbaren Wild. 2. Aufl., 215 S., 129 Abb., 22 Tab., Berlin und Hamburg (Parey).

Haller-Probst, M.S. (1997): Die Verbreitung der Reptilien in den Klimazonen der Erde unter Berücksichtigung känozoischer Vorkommen Europas. - Cour. Forsch.-Inst. Senckenberg, 203: 67 S., 83 Abb., 30 Tab., Frankfurt.

Haltenorth, T. (1959): Beiträge zur Kenntnis des mesopotamischen Damhirsches Cervus (Dama) 
mesopotamicus (BROOKE, 1875) und zur Stammesgeschichte allgemein. - Säugetierkdl. Mitt., Sonderheft, 7: 1-55, Stuttgart.

Hemmer, H. (1993): Felis silvestris Schreber, 1777 Wildkatze. - In: StubBE, M. \& KRAPP, F.: Handbuch der Säugetiere Europas, Raubsäuger - 2: 1076-1117, Wiesbaden.

Houben, C. (2001): Die Wirbeltiere aus der eemzeitlichen Fundstelle Lehringen (Niedersachsen, Deutschland). - Diplomarbeit, unveröff., 107 S., 7 Abb., 9 Taf., Bonn.

JACOB-Friesen, K.H. (1956): Eiszeitliche Elefantenjäger in der Lüneburger Heide. - Jb. Röm.-Germ. Zentralmuseum, 3: 1-22, Mainz.

Jakubowski, G., Krysiak, K. \& Roskosz, T. (1968): The Forest Elephant - Palaeoloxodon antiquus (FALC. \& CAut., 1847) from Warsaw, Prace Muzeum Ziemi, Prace Paleozoologiczne, 12: 187-212, Warschau.

Jessen, K. \& Milthers, V. (1928): Stratigraphical and palaeontological studies of interglacial freshwater deposits in Jütland and Northwest-Germany. - Danm. Geol. Unders., II. Raekke, 48: 1-379, Kopenhagen.

KeLlNER, M. (1986): Vergleichend morphologische Untersuchungen an Einzelknochen des postcranialen Skeletts in Europa vorkommender Ardeidae. - 222 S., Diss. Universität München.

Koenigswald, W.v. [Hrsg.] (1988): Zur Paläoklimatologie des letzten Interglazials im Nordteil der Oberrheinebene. - Paläoklimaforschung, 4: 327 S., 137 Abb., Stuttgart (Gustav Fischer).

- (1991): Exoten der Großsäuger-Fauna des letzten Interglazials von Mitteleuropa. - Eizeitalter u. Gegenwart, 41: 70-84, Hannover.

- (1999a): Palökologie und Vorkommen des pleistozänen Auerochsens (Bos primigenius Bojanus, 1827) im Vergleich zu den großen Rindern des Pleistozäns. - In: Weniger, G.-C. [Hrsg.]: Archäologie und Biologie des Auerochsens. - Wiss. Schriften des Neanderthal-Museums, 1: 23-33, Mettmann.

- (1999b): Hat der Mensch das Aussterben der großen pleistozänen Pflanzenfressser verursacht? - Kaupia, Darmstädter Beitr. zur Naturkd., 9: 193-201, Darmstadt.

- (2002): Lebendige Eiszeit. - 190 S., 198 Abb., Darmstadt (Wissenschaftliche Buchgesellschaft).

- \& Heinrich, W.-D. (1999): Mittelpleistozäne Säugetierfaunen aus Mitteleuropa - der Versuch einer biostratigraphischen Zuordnung. - Kaupia, Darmstädter Beitr. zur Naturkd., 9: 53- 112, Darmstadt.

KRÄUSEL, R. (1955): Die Interglazialflora von Lehringen. - Palaeontographica, B, 97: 47-73, 3 Abb., 1 Tab., Frankfurt.

LAmbrecht, K. (1933): Handbuch der Palaeornithologie. - 1024 S., 209 Abb., 4 Taf., Berlin (Borntraeger).
Lister, A.M. (1994): Evolution of the giant deer, Megaloceros gigenteus (BlUMENBACH). - Zool. J. Linnean Society, 112: 65-100, London.

LitT, T. (1994): Paläoökologie, Paläobotanik und Stratigraphie des Jungquartärs im nordmitteleuropäischen Tiefland. - Dissertationes Botanicae, 277: 185 S., 50 Abb., 10 Tab., 4 Beil., Berlin, Stuttgart.

- (2001): Waldland Mitteleuropa - Megaherbivoren aus paläobotanischer Sicht. - Ber. a. d. Bayr. Landesanst. Wald- u. Forstwirtsch., 27: 49-64, Freisingen.

MiнÁLIK, J. (1995). Der Wels. - N. Brehm-Bücherei, 209: 2. Aufl., 71 S., 52 Abb., Magdeburg (Westarp Wissenschaften).

Mlynarski, M. (1969): Fossile Schildkröten. - N. Brehm-Bücherei. - 396 S., Wittenberg (Ziemsen).

- \& Wermuth, H. (1971): Die Schildkröten. - In: GrizemeK , B. [Hrsg.]: Grizemeks Tierleben, 6, 3: 75-128, Zürich (Kindler).

Mưller, H. (1987): Beobachten und bestimmen - Fische Europas. - 320 S., Leipzig (Radebeul).

Pfeiffer, T. (1997): Die fossilen Damhirsche der Oberrheinebene im Vergleich zu Dama dama aus Neumark-Nord (Sachsen-Anhalt), Lehringen (Niedersachsen) und dem rezenten europäischen Damhirsch. - Z. Jagdwiss. 43: 221-239, Berlin.

- (1998): Capreolus suessenbornensis KAHLKE 1956 (Cervidae, Mammalia) aus den Mosbach-Sanden (Wiesbaden-Biebrich) mit einem Beitrag zur Stellung der Rehe im System pleistozäner und holozäner Cerviden. - Mainzer naturwiss. Archiv, 36: 47-76, 11 Abb., 1 Tab., Mainz.

- (1999): Sexualdimorphismus, Ontogenie und innerartliche Varibilität der pleistozänen Cervidenpopulation von Dama dama geiselana Pfeiffer 1998 und Cervus elaphus L. (Cervidae, Mammalia) aus Neumark-Nord (Sachsen-Anhalt, Deutschland). - Berliner geowiss. Abh., E, 30: 207-313, Berlin.

RAUfuB, I. (2001): Computergestützte Arealanalysen pleistozäner Säugetiervergesellschaftungen - Die Guilday Methode. - Diss., 122 S., 52 Abb., Universität Bonn.

Reichenau, W.v. (1912): Einiges über Schädel und Gebiß der Biber (Castoridae). - Jb. nass. Ver. Naturkd., 65: 208-226, Wiesbaden.

SELLE, W. (1962): Geologische und vegetationskundliche Untersuchungen an einigen wichtigen Vorkommen des letzten Interglazials in Norddeutschland. - Geol. Jb., 79: 295-352, 17 Abb., 11 Tab., 1 Taf., Hannover.

SickenberG, O. (1969): Die Säugetierfauna der Kalkmergel von Lehringen (Krs. Verden/Aller) im Rahmen der eemzeitlichen Faunen Nordwestdeutschlands. - Geol. Jb., 87: 551-564, 2 Abb., 2 Tab., Hannover. 
Staesche, K. (1941): Nashörner der Gattung Dicerorhinus aus dem Diluvium Württenbergs. - Abh. Reichsst. Bodenforsch., N.F., 200: 148 S., 2 Abb., 14 Taf., Berlin.

SteusLoff, U. (1954): Interglazialmollusken von Nedden-Averbergen im Kreis Verden. - N. Jb. Geol. Paläont., Abh., 100: 286-288, 1 Tab., Stuttgart.

StOller, J. (1918): Geologischer Führer durch die Lüneburger Heide. - 168 S., 38 Abb., 1 Kt., Braunschweig (Vieweg).

STRIEGLer, R. (1991): Die Europäische Sumpfschildkröte (Emys orbicularis) im Eem von Schönfeld. - Natur u. Landsch. Niederlausitz, Sonderheft, 1: 130-168, Cottbus.

Struckmann, C. (1884): Über die bisher in der Provinz Hannover aufgefundenen fossilen und subfossilien Reste quartärer Säugetiere. - Ber. naturhist. Ges. Hannover, 33/34: 3-36, Hannover.
STUART, A.J. (1976): The history of the mammal fauna during the Ipswichian/Last interglacial in England. Phil. Trans. roy. Soc. London, B, 276/945: 221-250, London.

Terofal, T. (1984). Süßwasserfische in europäischen Gewässern. - 288 S., 200 Abb., München (Mosaik).

Thieme, H. \& Veil, S. (1985): Neue Untersuchungen zum eemzeitlichen Elefanten-Jagdplatz Lehringen, Ldkr. Verden. - Die Kunde, N. F., 36: 11-58, Hannover.

Ullrich, H. (1978): Reptilienreste aus den jungpleistozänen Travertinen von Burgtonna in Thüringen. Quartärpaläont., 3: 97-102, Berlin.

- (1984): Sumpfschildkrötenreste aus dem jungpleistozänen Travertin von Weimar. - Quartärpaläont., 5: 325-344, Berlin.

VILCINSKAS, A. (1993): Einheimische Süßwasserfische. 207 S., Augsburg (Naturbuch Verlag). 